changes in white matter with sparing of scattered small areas consistent with a "tigroid pattern" of myelin preservation characteristic of PMD. (Shimomura $\mathrm{C}$ et al. Magnetic resonance imaging in Pelizaeus-Merzbacher disease. Pediatr Neurol $1988 ; \underline{4}: 124-5)$.

COMMINT. Described by Pelizaeus in 1885 and by Merzbacher in 1910, this heredofamilial disease transmitted as an X-linked recessive character and occurring chiefly in males is a slowly progressive leukodystrophy with a long course, patients not infrequently surviving into middle age. When the family history is negative, as in the above case, confirmation of the diagnosis during life is difficult but may be facilitated by the MRI findings and may permit appropriate genetic counseling.

\title{
CONGENITAL AMYELINATING NEUROPATHY
}

A case of severe neurogenic arthrogryposis multiplex congenita caused by absence of peripheral nerve myelin in an infant who died at age 31 days of aspiration pneumonia is described from the Dept of Neurology, Neuromuscular Division, Johns Hopkins University School of Medicine, Baltimore, MD. The infant, delivered by Cesarean section, had Apgar scores of one at 1 and 5 minutes, and examination revealed multiple fixed joints, small chin and triangular face, bilateral extraocular and facial pareses, diffuse hypotonia, muscle atrophy, and areflexia. Absence of myelin in the peripheral nerves at autopsy reflected an arrest in the differentiation or maturation of Schwann cells at the stages of elongation and longitudinal growth of the mesaxon. The authors refer to serial ultrasonography to assess fetal movement after the 18th week, the time of onset of peripheral nerve myelination, as an antenatal diagnostic technique in such families, and as suggested by Miskin M et al. (Charnas $\mathrm{L}$ et al. Congenital absence of peripheral myelin: Abnormal Schwann cell development causes lethal arthrogryposis multiplex congenita. Neurology June $1988 ; 38: 966-974)$.

Carmenr. The most common cause of arthrogryposis multiplex congenita is probably an amyoplasia due to anterior horn cell maldevelopment and associated muscle atrophy. Other causes include anterior horn cell degeneration, congenital myopathies, mechanical interference with fetal movement, and peripheral neuropathy. Paralysis of fetal movement is the common link in all forms of the disorder. For a comprehensive account of arthrogryposis, the reader should refer to Clinical Orthopedics April 1985;194, an issue devoted entirely to the topic. A long-term follow-up study showed that 17 of 34 patients examined at 16 years of age or older were able to walk independently and 9 others walked with the aid of crutches or braces. The prognosis is obviously hopeful in a majority of cases with nonprogressive underlying causes, and appropriate orthopedic procedures can achieve correction and relative independence at an early age.

\section{SEIZURE DISORDERS}

\section{POST-TRAUMATIC SEIZURES}

Factors influencing the occurrence of post-traumatic seizures in 92 of 937 children with head injuries (9.8\%) were studied in the Division of 